\title{
LA NATION RÉPUBLICAINE AU-DELÀ DES LANGUES PREMIÈRES
}

\section{THE REPUBLICAN NATION BEYOND FIRST LANGUAGES}

\author{
Aurélien Aramini \\ Université de Franche-Comté
}

\section{RÉSUMÉ}

Avec la Révolution française et sa politique de la langue, s'est posée de manière radicale la question des relations qu'entretiennent « langue » et « nation ». Cet article repère dans la mutation des discours français sur les « langues premières » de la Restauration à l'avènement de la Troisième République l'émergence d'une pensée républicaine de la nation pour laquelle l'appartenance politique n'est plus ordonnée à l'enquête ethnolinguistique. Dans les discours sur les « langues premières » de la Restauration à la Troisième République se lit une dynamique de rupture quant à la conception du lien entre communauté politique et langues premières : si des auteurs tels que Nodier ou Michelet trouvaient dans les langues des pères (les " patois ") ou dans celles des supposés ancêtres indo-européens les sources d'une régénération politique, la cité moderne qui s'esquisse en creux dans La Cité antique de Fustel et l'identité nationale que défend Renan contre Strauss ne plongent leurs racines ni dans l'antiquité indo-européenne ni dans les profondeurs du terroir dialectal. Se dessine alors une certaine conception de la nation pour laquelle ne fait plus sens l'ancrage dans les mondes humains sédimentés dans les langues premières.

Mots clefs : Langue, patois, nation, indo-européen, république

\section{ABSTRACT}

With the advent of the French Revolution and its politics of language, people began to radically question the relationships between "language" and "nation." This article identifies the emergence of a republican conception of the nation within the transformation of the French discourse on "first languages" 
from the Bourbon Restoration to the ascension of the Third Republic, in which political affiliations are no longer tied to ethnolinguistic enquiries. In the discourse during the period between the Bourbon Restoration and the Third Republic, one can glimpse a rupture of the tie between the ideas of political community and first languages: if authors such as Nodier or Michelet saw the languages of fathers ("patois") or those of supposed Indo-European ancestors as the sources of a political regeneration, the modern city which is sketched in hollow in The ancient City of Fustel and the national identity that Renan defended to Strauss find their roots neither in Indo-European antiquity nor in the depths of dialectical territories. We find, then, a certain conception of the nation for which an entrenchment in first languages sedimented in human worlds no longer makes sense.

Key words: Language, patois, nation, Indo-European, republic

\section{RESUM}

\section{La nació republicana més enllà de les primeres llengües}

Amb la Revolució Francesa i la seva política lingüística va sorgir radicalment la qüestió de la relació que s'estableix entre "llenguatge" i "nació". Aquest article es centra en la mutació del discurs francès sobre les "llengües primeres" de la Restauració durant l'adveniment de la Tercera República amb el sorgiment del pensament republicà de la nació per al que la filiació política no es fonamenta des d'un plantejament etnolingüístic. Als discursos sobre les "llengües primeres" de la Restauració de la Tercera República es llegeix una ruptura dinàmica en el disseny de l'enllaç entre la comunitat política i els primers idiomes: si autors com Nodier o Michelet trobaven en les llengües dels pares (el "patois") o en les dels suposats avantpassats indoeuropeus les fonts d'una regeneració política, la ciutat moderna llurs diferències amb la ciutat antiga queden esbossades a La Ciutat antiga de Fustel i la identitat nacional que Renan defensa front a Strauss no enfonsen els seus arrels en l'antiguitat indoeuropea ni en les profunditats del dialecte local. A partir d'ací es dibuixa una certa concepció de la nació per als que ja no té sentit la fixació sobre els mons humans sedimentats en les llengües primeres.

Paraules clau: llengua, patois, nació, indoeuropea, república 
La question des relations qu'entretiennent la « langue » et la «nation » s'est posée de manière radicale avec la Révolution française dès lors que la langue est devenue un problème politique central. Au XIXe siècle, se réalise, sur le plan social, l'homogénéisation linguistique de la France en raison des " convergences des facteurs de démocratisation aboutissant à l'institution du français scolaire d'école primaire dans les années 1880 ».' Toutefois, parallèlement à ce mouvement d'uniformisation linguistique, ce siècle témoigne d'une conscience aiguë du "foisonnement » des langues de la France $i^{2}$ il se passionne aussi pour la philologie comparée qui inscrit le «français » dans une histoire longue des langues indo-européennes conduisant jusqu'aux contreforts de l'Himalaya. Dès les premières décennies du siècle historien, émergent des discours, à la confluence de la philologie, de I'histoire, de la philosophie et de la politique, portant sur deux ensembles de langues qui précèdent ou dont procède la langue française : I'ensemble des langues premières de la France et celui des langues de l'antiquité indo-européenne. Or, dans le cadre du profond renouvellement de l'étude des «langues premières » dans le sillage de l'historiographie romantique et de la philologie indo-européenne, la langue, loin de n'être qu'un système de signes, est alors conçue comme l'expression de l'esprit et de la volonté $d^{\prime}$ un peuple ${ }^{3}$ voire, comme l'écrit Michelet dans son Histoire romaine, «le monument le plus important de son histoire » et le critère grâce auquel ce peuple « se classe dans telle ou telle division de l'espèce humaine $»{ }^{4} \mathrm{Si}$

1 BALIBAR, Renée (1985), L'Institution du français Essai sur le colinguisme des Carolingiens à la République, Paris, PUF, coll. "Pratiques théoriques», p. 13.

2 Sur la situation linguistique de la France au XIX siècle où les langues foisonnent, voir l'ouvrage de WEBER, Eugen (1983), La fin des terroirs La modernisation de la France rurale 1870-1914, Paris, Fayard, tout particulièrement le sixième chapitre intitulé « Des langues à foison ».

3 Sur ce point, voir l'analyse de Foucault relative à la philologie dans FOUCAULT, Michel (1966), Les mots et les choses Une archéologie des sciences humaines, Éditions Gallimard, coll. Tel, pp. 302-303 sqq.

4 MICHELET, Jules (1971), Histoire romaine, OEuvres complètes, publiées sous la direction de Paul Viallaneix, Paris, Flammarion, t. II, p. 359. 
les langues sont désormais conçues comme les plus anciens monuments de l'histoire des peuples et l'expression de leur volonté la plus propre, les langues premières ne pouvaient-elles pas fournir les pierres de fondation de l'édification de la nation?

Si une réponse affirmative est lisible dans des discours libéraux ou républicains des deux premiers tiers du XIXe siècle, chez des auteurs tels qu'Augustin Thierry, Charles Nodier ou encore Michelet, c'est bien plutôt une réponse négative qui s'impose sous la plume d'auteurs emblématiques de la Troisième République comme Fustel de Coulanges, Renan ou Renouvier. L'étude des discours sur les langues " premières $»^{5}$ qu'elles soient celles de la France ou de l'Orient indo-européen révèle que ces discours ne présentent pas un caractère théorique homogène durant la période comprise entre la Restauration et l'avènement de la Troisième République. Ils donnent à voir des conceptions parfois diamétralement opposées concernant la nature de ses langues premières, concernant la valeur et la signification que peuvent avoir pour nous autres modernes les mondes humains et les traditions que prétendent reconstruire à partir de l'étude de ces langues premières certains philologues d'avant le tournant linguistique saussurien. C'est en raison de leur hétérogénéité que les discours sur les langues premières de la Restauration à l'avènement de la Troisième République fournissent un champ d'étude privilégié pour mettre en lumière les problèmes théoriques, les tensions conceptuelles et les conflits politiques où s'inscrit la dynamique

5 Dans la sociolinguistique contemporaine, l'expression « langue première » renvoie à la langue d'origine ou à la langue maternelle par opposition à la langue acquise par l'enfant au sein d'institutions telles que l'école par exemple. Ce n'est pas directement ce sens donné à la langue première que j'adopte. Dans la présente étude, je privilégierai un double usage de cette expression repérable au XIXe siècle. La langue « première » désigne alors la première langue, au sens chronologique, de l'humanité ou les premières langues parlées dans les premières sociétés humaines (cf. par exemple DELAMÉTHERIE Jean-Claude (1806), Considérations sur les êtres organisés : de la perfectibilité et de la dégénérescence des êtres organisés, Paris, Courcier, $\mathrm{p}$. 207 sqq.). Elle peut aussi qualifier les langues dont la présence est attestée avant la constitution d'une langue moderne : ainsi le « celte » ou le « gaël » vis-à-vis de la langue française (cf. par exemple EDWARDS, William Frederic (1844), Recherches sur les langues celtiques, Paris, Imprimerie Royale, p. 80 sqq). Renouvier utilise l'expression «langue première » en 1869 dans la Science de la morale voir infra. Par « langues », j'entends " tout système d'expression servant à communiquer dans un groupe social »; la distinction entre « langue », " dialecte » et " patois » n'exprimant que « des jugements de valeur et des critères politiques » (ces formules sont de Sonia Branca-Rosoff dans l'article BRANCA-ROSOFF, Sonia (1999) « Normes et dialectes » in AUROUX, Sylvain (dir.), Histoire des idées linguistiques, t. III « L'hégémonie du comparatisme », Mardaga coll. «Philosophie et langage », pp. 45-53. 
conduisant à l'élaboration d'une conception républicaine du social et de la nation. Cette dynamique de rupture est particulièrement manifeste dans les réflexions sur les langues premières développées, dans la décennie 1860/1870, par des historiens comme Fustel de Coulanges ou par des philosophes comme Renouvier ou Renan. Car ces réflexions expriment, selon des modalités différentes, une même exigence, celle de faire advenir une pensée du social et de la nation neutralisant deux tentations auxquelles de nombreux discours sur les langues premières ont pu céder durant les deux premiers tiers du XIXe siècle : d'une part, la tentation du particularisme qui réduit l'homme à une origine ethnique et géographique en deçà de la nation, précédant l'institution républicaine du citoyen ; d'autre part, la tentation de l'ethnodifférentialisme qui distingue dans l'histoire universelle, différentes traditions humaines inconciliables à partir de la diversité des familles de langues - indo-européennes et sémitiques - et qui prétend fournir un principe régénérateur à la société de l'avenir. Ce double refus conduit à une pensée de la nation pour laquelle l'appartenance politique n'est plus ordonnée à l'enquête ethnolinguistique.

\section{Les langues premières de la France}

\section{La Révolution et les langues premières}

II parait indéniable que la Révolution française avec sa politique de la langue est l'un des jalons essentiels de l'histoire de l'institutionnalisation du français ainsi que l'ont montré Certeau, Julia et Revel dans un ouvrage désormais classique. ${ }^{6} \mathrm{Au} X \mathrm{XI} \mathrm{X}^{\mathrm{e}}$ siècle, la question des langues premières de la France se pose dans la perspective historique nouvelle ouverte par la Révolution française et par la politique de la langue qu'ont initiée les rapports de Barère et de l'abbé Grégoire afin de créer des mœurs républicaines en détruisant les patois - " derniers vestiges de la féodalité détruite $»^{7}-$ la diversité linguistique étant conçue comme un état babélien de confusion empêchant que "nos cœurs » puissent être à " I'unisson »..$^{8}$ Véritable trait $d^{\prime}$ union entre l'entreprise révolutionnaire jacobine et la politique libérale de la Monarchie de Juillet, le grand Carnot soutiendra pendant les Cent Jours la création d'une société savante qui aura un rôle décisif dans l'entreprise d'unification linguistique : la Société pour l'instruction élémentaire, dont des

6 CERTEAU, Michel de, JULIA, Dominique et REVEL, Jacques (2002), Une Politique de la langue, Éditions Gallimard, collection «folio histoire », première édition 1975.

7 Ibid., p. 347.

8 lbid., p. 339. 
membres « font déjà partie de la Société d'encouragement pour l'Industrie nationale ». ${ }^{9}$ Par ce biais, le projet révolutionnaire jacobin va rencontrer le projet politique libéral dont le représentant majeur n'est autre que Guizot, I'auteur de la loi de 1833 sur l'enseignement primaire. ${ }^{10}$

Aux origines de la République - entre 1789 et 1795 - il s'avère cependant que Révolution et monolinguisme ne s'impliquent pas mutuellement, bien au contraire : "les tout premiers temps de la Révolution connaissent en effet une courte période de redécouverte du plurilinguisme en France. Nombre de textes politiques, notamment des lois et décrets, sont traduits du français en d'autres idiomes ". " Davantage encore, on ne saurait nier l'existence de textes de facture jacobine en langue locale. ${ }^{12} \mathrm{C}^{\prime}$ est seulement par la suite que le rapport aux langues premières va être progressivement associé à une certaine conception de la nation, au prix d'une relecture de la Révolution française selon le schéma faisant correspondre le plurilinguisme au fédéralisme et le monolinguisme au centralisme jacobin. Même si cette lecture de la relation entre Révolution française et pluralisme linguistique est simplificatrice et si l'identification entre nation et langue ne va pas de soi au cours du siècle, ${ }^{13}$ l'émergence d'une langue républicaine s'imposant dans l'espace national est indéniable. Pour autant, dans les perspectives ouvertes par Jacques Guilhaumou, ${ }^{14}$ il est légitime de revenir aux sources de la relation conflictuelle entre République et diversité linguistique en s'in-

9 BALIBAR, L'institution du français, p. 252.

10 Concernant le rapport entre la Révolution française et les langues régionales, voir l'article de SCHLIEBEN-LANGE, Brigitte (1999), "La Révolution française », première section du premier chapitre "Luttes sociales, dialectes, contraintes », in AUROUX, Histoire des idées linguistiques, pp. 23-34. Pour une étude de la politique de la langue lors de la Révolution française, je renvoie à l'ouvrage Une Politique de la langue, op. cit. Toutefois, l'identification entre monolinguisme et jacobinisme doit être nuancée et présentée comme une certaine lecture de la Révolution française, voir infra les travaux de Jacques Guilhaumou.

11 PLÖTNER, Bärbel (1994), «Langue littérature et identités nationales et régionales Jacob Grimm entre la France et l'Allemagne Le cas breton » in Espagne, Michel et Werner Michael (dir.), Qu'est-ce qu'une littérature nationale? Approche pour une théorie interculturelle du champ littéraire, Paris, Éditions de la Maison des sciences de l'homme, (pp. 212-234), p. 215.

12 Voir GUILHAUMOU, Jacques, (1989) : La langue politique et la Révolution française. De l'événement à la raison linguistique, Paris, Méridiens Klincksieck.

13 Fustel de Coulanges le rappelle avec force à Mommsen dans sa lettre L'Alsace est-elle allemande ou française : réponse à $M$. Mommsen, publiée dans la Revue des deux mondes en octobre 1870.

14 Voir tout particulièrement l'article : GUILHAUMOU, Jacques (1986) : « Les Jacobins et la langue provençale : I'initiative linguistique des "missionnaires patriotes" marseillais en 1792 », $\mathrm{Ca}$ hiers critiques du patrimoine, Obradors occitan en Provença, 2, p. 11-124. 
téressant tout particulièrement aux discours républicains concernant les langues premières de la France dont la présence est attestée, sur le territoire national, avant la constitution de la langue française et dont celle-ci porte éventuellement la trace.

Contre cette dynamique " monolinguiste » qui aboutira à "l'institution du français » sous la Troisième République, se formulent des défenses des langues locales mobilisant des stratégies argumentatives différentes, parfois contradictoires. Cependant, ces positionnements très divers entretiennent tous des relations privilégiées avec l'historiographie telle qu'elle sera renouvelée par Augustin Thierry durant la décennie 1820/1830 parce qu'il confère un sens historique aux langues régionales bien différent de celui que leur ont accordé Barère ou Grégoire.

\section{Le sens historique des "patois"}

Dans les Lettres sur l'histoire de France, c'est par le biais de la philologie qu'Augustin Thierry met au jour le fait historique fondateur de l'histoire de France, à savoir la conquête par les Francs - barbares, étrangers et nomades - du territoire occupé par des peuples indigènes de la Gaule romaine. C'est en exhumant les noms originaux des premiers rois de France - Hlode-wig et non Clovis - qu'il construit l'idée d'une lutte des races qui aurait fracturé la société et rejeté dans les marges de l'histoire les peuples vaincus. Comme l'histoire officielle a réduit l'histoire du royaume à celle des grandes familles issues des conquérants, l'historien libéral a l'intuition que l'existence des peuples vaincus peut encore se lire dans l'épaisseur linguistique de la France. ${ }^{15}$ Dans les replis des dialectes et des patois, se serait conservée l'histoire de ceux qui n'ont pas d'histoire : même si l'esprit de la conquête a dépossédé les vaincus, les langues constituent la trace de leur existence et de la lutte des races. Par le biais de l'histoire des langues, Augustin Thierry appelle à étudier les contre-histoires locales restées dans l'ombre de l'histoire. ${ }^{16}$ Avec l'historien romantique, s'amorce une histoire du peuple ou plutôt " des peuples », dont la présence infranationale est

15 Je me permets de renvoyer à mon article ARAMINI, Aurélien (2014), "L'Archéologie linguistique du pouvoir et du peuple chez Augustin Thierry », Revue d'histoire du XIXe siècle, $n^{\circ} 49$, 2014/2, pp. 177-191.

16 La lettre VIII des Lettres sur I'histoire de France formule les raisons historiographiques et politiques de l'intérêt d'Augustin Thierry pour « les dialectes et les patois provinciaux » (THIERRY, Augustin (1827), Lettres sur I'histoire de France, Paris, Sautelet, Ponthieu et compagnie, 1827, lettre VIII, p. 81). 
attestée par les langues vernaculaires qui foisonnent dans la France de la Restauration. ${ }^{17}$ Dès lors, les langues « locales » ne sont plus les débris de la féodalité passée mais "les traces vivantes ${ }^{18}$ des peuples vaincus et l'expression de la résistance toujours actuelle contre l'esprit de la conquête qui structure la société française depuis les invasions barbares.

Les travaux publiés à partir de la Restauration sur le bas breton, ${ }^{19}$ le basque ${ }^{20}$ les « patois » de l'Est de la France ${ }^{21}$ et, plus tard, sur le celte ${ }^{22}$ et les langues romanes, ${ }^{23} s^{\prime}$ appuient bien souvent sur un discours mobilisant la thématique de la conquête ou celle de la lutte des races. Certes, le propos d'Augustin Thierry a indéniablement nourri des formes historiographiques très éloignées des perspectives politiques libérales qui étaient les siennes sous la Restauration tel que le « mouvement nationalitaire occitan ». ${ }^{24}$ Mais, quelles qu'aient pu être les mobilisations politiques de l'historiographie d'Augustin Thierry, il $n^{\prime}$ en demeure pas moins que la langue n'est plus considérée chez lui comme un « système de signes » : elle devient l'expression même d'un peuple doué d'une consistance historique.

\section{Le patois comme " langue du père "}

Parmi les textes de la défense des «langues premières » de la France repérable entre la Restauration et l'avènement de la Troisième République, l'analyse philosophique des "patois » développée par Charles Nodier dans ses Notions élémentaires de linguistique ${ }^{25}$ possède les caractéristiques essentielles $d$ 'une défense des « langues premières » mobilisant les catégories de l'historiographie romantique. Au treizième chapitre de cet ouvrage publié en $1834^{26}$ il propose une défense qui procède en deux temps. ॥

17 Voir WEBER, La fin des terroirs...

18 THIERRY, Augustin (1825), Histoire de la Conquête de l'Angleterre par les Normands, Paris, Firmin Didot, 3 vol., t. I, pp. I.

19 Voir LE GONIDEC, Jean-François, (1821), Dictionnaire breton-français, Angoulême.

20 Voir LÉCLUSE, Fleury de (1826), Grammaire basque, Toulouse.

21 Voir FALLOT, Samuel (1828), Recherches sur le patois de Franche-Comté, de Lorraine et d'Alsace, Montbéliard, Deckherr.

22 Voir PICTET, Adolphe (1837), De l'affinité des langues celtiques avec le sanscrit, Paris, Duprat.

23 Voir BRUCE-WHYTE, A. (1841), Histoire des langues romanes et de leur littérature depuis leur origine jusqu'au XIVe siècle en trois tomes, Paris, Treuttel et Würtz.

24 Voir ARAMINI, «L'Archéologie linguistique du pouvoir et du peuple chez Augustin Thierry ».

25 NODIER, Charles, (1934), Notions élémentaires de linguistique, Paris, Renduel, voir tout particulièrement le chapitre XIII.

26 NODIER, «Des patois », Notions élémentaires de linguistique. 
construit d'abord une conception philosophique de ce qu'est un " patois » pour formuler ensuite les raisons pour lesquelles il n'est pas légitime de vouloir les détruire.

Nodier définit un patois comme étant la langue du "père ", du "pays ", de la «patrie ». Il précise qu'il ne traite pas des langues telles que le « bas breton » ou le "basque » qui " révèlent une nationalité individuelle, une autre origine et un autre génie ». ${ }^{27} \|$ est donc bien question des langues que les «lettrés » considèrent comme un « jargon confus et sans règles ». Deux points sont à examiner : le patois comme «langue » d'abord; et comme langue « du père » ensuite. D'emblée, Nodier envisage le patois comme une langue à part entière.

Sait-on seulement ce que c'est qu'une langue, et quelles profondes racines elle a dans le génie d'un peuple, et quelles touchantes harmonies elle a dans ses sentiments? Sait-on qu'une langue, c'est un peuple, et quelque chose de plus qu'un peuple, c'est-à-dire son intelligence et son âme ?228

Deux points sont ici à souligner : "la langue c'est un peuple», écrit-il d'abord. La thèse n'est pas originale, on la trouve déjà chez les Idéologues : la langue donne à voir les pratiques originales d'un peuple. Mais l'essentiel réside dans le second point qu'il ajoute immédiatement : " c'est quelque chose de plus qu'un peuple ». Quel est ce «plus»? Ce " plus» qu'un peuple, c'est ce qui fait que le peuple est peuple : c'est la langue qui lui donne l'âme sans laquelle il n'y aurait qu'un agrégat d'individus. La langue est l'âme qui fait tenir le corps du peuple.

La formule «langue du père " place immédiatement la question des patois dans la perspective diachronique du fait « linguistique ». Dans le présent des langues de la France coexisteraient ainsi deux temporalités distinctes: celle de la langue "moderne » et celle de langues qui sont la permanence, dans le présent, d'un état de la langue qui précède chronologiquement mais aussi logiquement la langue moderne. Le patois est la langue du père qui a perduré. Qui est ce « père » dont le patois est la langue? C'est dans les termes de l'historiographie d'Augustin Thierry que répond Nodier : le patois est la langue des "races simples » qui précèdent le mouvement historique de centralisation et les progrès de la civilisation. Le patois est 
la langue de races "éloignées du centre, isolées, par des circonstances que je tiens pour extrêmement heureuses, des moteurs immédiats de l'éducation progressive ». ${ }^{29}$ Les "patois " sont donc les traces vivantes des « races » qui ont résisté à la centralisation et qui sont restées en marge du progrès. Comme l'entendait Augustin Thierry, ils sont les « témoins » d'une vie « simple » qui précède l'histoire. Mais attention, ici le terme « simple » n'est pas synonyme de "primitif », il désigne ce qui est au plus près du sens originaire du monde car le patois ne s'est « modifi[é] que très lentement » et il « conserve le mot de la manière dont le mot s'est fait ». ${ }^{30}$ Nodier donne alors la formule philosophique du « patois »: «c'est la langue native, la langue vivante et nue $\gg .^{31}$

Les langues régionales sont donc conceptuellement pensées comme « premières » non seulement chronologiquement mais aussi $d^{\prime} u n$ point de vue gnoséologique. Elles sont révélatrices d'un mode d'être qui précède la civilisation et exprime un rapport originaire au monde dont la langue moderne s'est éloignée. La langue locale est la sédimentation d'une expérience archaïque du foyer, porteuse d'une vision d'un monde qui précède le découpage de la nature selon les catégories de la raison opératoire qui s'est éloignée du sens du monde.

La défense construite par Nodier repose sur l'affirmation de la « liberté du langage ». Détruire les patois, ce serait remettre en cause cette liberté. La liberté est alors conçue comme l'affirmation d'une différence, ici en l'occurrence de la différence du " père »: la liberté du langage signifie donc la reconnaissance de la particularité de ma filiation comme sujet parlant. C'est au nom de la revendication de cette différence de filiation que Nodier élabore une stratégie argumentative consistant à mettre en lumière les conséquences auxquelles conduit la volonté de détruire les patois : « quand on en est venu à de pareilles théories, il faut avoir au moins l'affreux courage d'en adopter les conséquences. II faut anéantir les villages avec le feu; il faut exterminer les habitants avec le fer $\gg{ }^{32}$ La volonté d'uniformisation linguistique participe d'un projet de conquête. Selon cette lecture, l'anéantissement des patois s'inscrit donc contre le projet libéral dont l'historiographie - d'Augustin Thierry et même de Guizot - a dénoncé la destruction des libertés municipales des peuples gallo-romains par les hordes barbares. Nodier 
réactive ce schème de la " conquête » en faisant des partisans de l'uniformisation linguistique de nouveaux « barbares » conquérants. Il est intéressant de constater que Paul Lorrain, ardent défenseur du monolinguisme, proche collaborateur de Guizot et futur recteur de Lyon sous la monarchie de Juillet, utilise et revendique cette référence à la conquête dans son Tableau de l'instruction primaire en France : « [...] dût-on nous traiter de Vandale, nous sommes d'avis qu'on tranche au vif dans cette antique transmission des patois, et que chaque école soit une colonie de la langue française en pays conquis. ». ${ }^{33}$ Ainsi, Nodier n'a pas tort d'inscrire la négation de la « liberté du langage » dans la continuité d'une histoire agonistique qui a fracturé l'histoire de la France depuis quinze siècles. Cette nouvelle conquête - linguistique cette fois et non plus seulement territoriale - reproduira la conquête qui priva les Gallo-Romains de leurs libertés municipales à l'aube du Moyen-Âge et détruisit leur mode d'être dont les patois sont l'ultime témoignage. Dans le discours de Nodier, le monolinguisme signifie la mise à mort de la voix des pères où s'est sédimentée l'expérience originaire du monde que la civilisation a perdue. La destruction des patois détruit une liberté qui n'est pas une liberté individuelle mais l'affirmation de la particularité de ma filiation linguistique, affirmation qui implique l'existence d'un "foyer associatif populaire et constitué » dont l'antiquité est attestée par le caractère " premier » de la langue et qui possède une identité propre précédant la nation. En défendant le « patois », je défends l'âme du peuple qui, d'une part, façonne mon identité particulière de sujet parlant et, $d^{\prime}$ 'autre part, donne sens à mon monde. ${ }^{34}$

\section{Les langues de l'Orient indo-européen chez Michelet À la recherche de l'origine : la révolution aryaniste}

Si l'étude des langues premières de la France s'inscrit dans le sillage de la Révolution française, l'étude des langues premières de l'ancien Orient prend son impulsion dans l'autre grand bouleversement qui modifia en profondeur les représentations de l'histoire humaine à l'aube du siècle de I'histoire, à savoir la « renaissance orientale » selon la formule d'Edgar

33 Cette phrase se trouve sous la plume de Paul Lorrain - qui deviendra sous la Monarchie de Juillet recteur de Lyon - dans LORRAIN Paul (1837), Tableau de l'instruction primaire en France, Paris, Hachette.

34 Voici la définition de la « linguistique » par Nodier : «simple histoire de la parole et de l'écriture considérées depuis leur origine jusqu'à la fin de leurs premiers développements naturels » (NODIER, Notions élémentaires de linguistique). 
Quinet. ${ }^{35}$ En effet, durant les premières décennies du XIXe siècle, le déchiffrement des hiéroglyphes, du sanscrit védique, du pâli ou du zend a ajouté " trente siècles [...] à l'antiquité ${ }^{36}$ et fait surgir " plusieurs mondes oubliés qui reviennent juger celui-ci $» .^{37}$ Le déchiffrement du sanscrit renouvelle de manière décisive la philologie. Mais, dans le cadre de mon propos, je $\mathrm{m}^{\prime}$ attacherai moins à la révolution sanscriste ${ }^{38}$ qu'à la thèse " aryaniste » qui ne trouve une assise scientifique qu'en 1833 dans le Commentaire sur le Yaçna d'Eugène Burnouf. ${ }^{39}$

Dans cet ouvrage, l'idée d'un «fonds commun » des langues indo-européennes va être construite linguistiquement à partir d'une étude du " radical » des mots du vocabulaire «zend » : contre une lecture faisant du sanscrit la langue mère des idiomes indo-européens et de l'Inde le point de départ des migrations des peuples parlant ces langues, Eugène Burnouf émet l'hypothèse que le latin, le grec et le sanscrit classique sont les rejetons $d^{\prime}$ une langue mère « aryenne $»^{40}$ dont le sanscrit védique et le «zend »,

35 Reprise en 1842 dans le Génie des Religions, la formule est, à l'origine, le titre d'un article publié en 1841 dans la Revue des deux Mondes, t. 28, quatrième série, Paris, Bureau de la Revue des deux Mondes, 1841 (p. 112 sqq.). Pour une lecture critique de la «Renaissance orientale », cf. DEMOULE, Jean-Paul (2014), Mais où sont passés les Indo-européens?, Paris, Seuil.

36 MICHELET, Jules (1864), Bible de l'humanité, Paris, Chamerot, p. 484.

37 lbid.

38 Concernant la pertinence de l'usage du concept de « révolution » pour qualifier la découverte de la parenté du sanscrit et des langues européennes ainsi que la naissance de la grammaire comparée, je renvoie au premier chapitre de l'ouvrage de RABAULT-FEUERHAHN, Pascale (2008), L'archive des origines. Sanskrit, philologie, anthropologie dans l'Allemagne du XIX siècle, Paris, Cerf, p. 35 sqq. Je désigne par « sanscristes » les travaux linguistiques faisant du sanscrit la «souche » des langues indo-européennes (le terme "sanscriste » est utilisé au XIX siècle pour désigner les orientalistes spécialisés dans l'étude du sanscrit).

39 La lecture du Commentaire sur le Yaçna et des Études sur la langue et les textes zends (deux tomes, Paris, Imprimerie nationale, 1840-1850) engage une discussion des travaux de OLENDER, Maurice (1989), les Langues du paradis, Aryens et sémites : un couple providentiel, Éditions du Seuil, coll. "Hautes Études ». Concernant les enjeux politiques et philosophiques de l'œuvre d'Eugène Burnouf, je me permets de renvoyer à mon article ARAMINI, Aurélien (2014), "Zoroastre républicain Les enjeux philosophiques et politiques de la découverte des textes "zends" : Eugène Burnouf et Jean Reynaud », Klesis, 30/2014, pp. 27-54.

40 I| faut bien distinguer le terme « aryen » (écrit parfois « arien ») - figurant déjà chez Anquetil-Duperron et désignant un peuple ainsi nommé dès l'Antiquité grecque - et le concept linguistique $d^{\prime}$ « aryen » qui renvoie à un peuple hypothétique parlant une langue appelée « aryenne » dont les idiomes indo-européens constituent des rejetons. Christian Lassen serait le premier à utiliser le terme en ce dernier sens (LASSEN, Christian (1830), « Über Herrn Professor Bopps gram- 
langues plus archaïques que le sanscrit classique, sont demeurées les langues les plus proches. Par le biais d'une étude ethnolinguistique du Zend Avesta comparé aux Védas, Burnouf est conduit à localiser précisément le

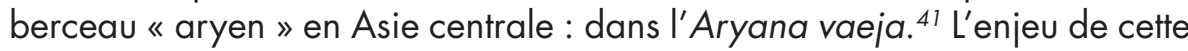
découverte est double pour Eugène Burnouf car la philologie ne se réduit pas à l'étude des mots. D'abord, elle permet d'écrire l'histoire archaïque des migrations humaines, longtemps « rejetée dans un passé impénétrable, parce qu'il échappe aux souvenirs de l'histoire ». ${ }^{42}$ Ensuite, elle éclaire I'histoire de l'esprit humain dont les textes zends et védiques donnent à voir l'expression originaire et les intuitions fondatrices. Si le propos d'Eugène Burnouf demeure dans une perspective universaliste en 1833 - inspiré par le concept $d$ '« universel fantastique » de Vico - le discours aryaniste s'oriente très rapidement, et tout particulièrement chez le premier Renan, dans une voie ethnodifférentialiste. Loin de traiter des structures de l'esprit comme certaines déclarations d'Eugène Burnouf auraient pu le donner à penser, les études indo-européennes vont contribuer à construire l'opposition entre deux « esprits », l'esprit sémitique et l'esprit aryen en s'appuyant sur l'existence « antéhistorique » d'un peuple «Aryâ » ou « indo-européen » censé avoir réellement vécu dans une région précise et dont les idées et les mœurs, établies à partir de la comparaison des mots, constitueront le fonds commun de la culture des futurs peuples indo-européens. Cette orientation est particulièrement manifeste dans la mobilisation du discours philologique aryaniste que fera Michelet dans la Bible de l'humanité.

\section{La linguistique aryaniste et son influence sur la pensée sociale}

En 1864, le problème de Michelet est de donner un credo fondateur à la République à venir, un credo sensé dynamiser les cœurs afin de leur faire vouloir la fraternité. Or, ce sont les traditions millénaires exhumées par les philologues que la Bible de l'humanité convoque pour ériger un nouvel autel domestique, pierre angulaire d'une régénération de la famille.

matisches System der Sanskrit-Sprache », Indische Bibliothek III, ed. August Wilhelm Schlegel, Bonn, 1830, pp. 1-113.) selon Konrad Koerner (KOERNER, Konrad (1989), "Observations of the Sources, Transmission, and Meaning of 'Indo-European' and related Terms in the Development of Linguistics" in KOERNER, Konrad (ed.) Practicing Linguistic Historiography, Amsterdam \& Philadelphia, John Benjamins Publishing Company, pp. 153-180, p. 169).

41 Eugène Burnouf établit que l'ancienne « Arie » correspond à la zone comprise dans le triangle dont les sommets sont, au nord, la « Sogdiane », à l'ouest, l'« Hyrcanie », au sud, l'« Arachosie ».

42 BURNOUF, Eugène (1833), " De la langue et de la littérature sanscrite », Revue des deux mondes, livraison du 1 er février 1833, p. 3-4. 
La Bible de l'humanité fonctionne à partir d'une lecture dichotomique de I'histoire universelle qui trouve chez Michelet sa première formulation en 1847 dans l'introduction de l'Histoire de la Révolution française : il a alors renoncé à une conception de l'histoire universelle comme une succession linéaire de civilisations, allant du primitif au civilisé (de l'Inde à la France), lecture qu'il adoptait en 1831. L'histoire telle qu'il l'envisage en 1847 se trouve fracturée en deux courants opposés et inconciliables : la Justice contre la Grâce. Sont alors posées de manière agonistique, deux séries d'identité : monarchie $=$ grâce $=$ christianisme $/$ république $=$ justice $=$ Révolution. En effet, tout le problème reste de donner une tradition religieuse à la Révolution française car telle est, pour Michelet, la cause profonde de son échec. L'acte théorique de la Bible de l'humanité consiste à incarner cette fracture de l'histoire entre la tradition de la Justice et la tradition de la Grâce dans des peuples historiques et de mettre au jour une tradition religieuse radicalement non chrétienne de la Justice. En mobilisant les ressources des textes des philologues, Michelet pose d'abord la tradition de Justice supposée naître chez les peuples issus des Aryâs primitifs : Indiens, Perses, Grecs. Ils furent créateurs des types vitaux de la société : famille (justice mutuelle entre l'homme et la femme), travail (justice distributive entre l'homme et la nature), éducation. Le principe générateur de ces types vitaux réside dans l'attention que ces peuples portent à la « nature » et qui s'exprime dans leur vision polythéiste (repérable dans le culte du feu au sein de la famille = Védas, dans l'affirmation de la fraternité avec le monde animal = Ramâyanâ, l'attention à la terre pour l'agriculture et l'élevage dans le Zend Avesta, la croyance en la De-meter Terra Mater dans la Grèce archaïque). À ces peuples créateurs sont opposés les peuples sémitiques - la tradition syro juive - qui inventèrent la "Grâce », l'idée d'un Dieu qui sauve «sans raison » : ces peuples auraient une autre conception de la famille (tyran et vierge), nieraient le travail au profit de la spéculation et, au lieu d'éduquer, seraient dans l'attente du miracle. Et ces dimensions convergent dans le «monothéisme » sémitique, religion antinaturaliste qui expulse la nature et qui est à la fois cause et effet d'un mode d'être contre-nature. Reprenant les thèses de Renan, Michelet met en place une perspective ethnodifférentialiste de l'histoire universelle : monde sémitique $=$ Grâce $=$ monothéisme = antinaturaliste $/$ monde aryen $=$ polythéisme $=$ naturalisme cosmothéiste = Justice. Le soubassement de ces équivalences apparaît chez Renan qui s'appuie sur des considérations purement linguistiques :

La nature, dans un tel système [le système de la langue sémitique], ne pouvait être conçue que comme absolument inanimée. Au lieu de cette nature vivante qui parla si puissamment à l'imagination des ancêtres de 
la race indo-européenne, ici c'est Dieu qui fait tout, en vue d'un plan connu de lui seul. ${ }^{43}$

Le cœur de l'argument de Renan repris par Michelet consiste à penser les formes élémentaires de la vie religieuse à partir de l'opposition entre monothéisme antinaturaliste et polythéisme cosmothéiste ; chez Renan, il apparaît que les formes élémentaires de la croyance ne sont pas seulement issues du social lui-même, mais que le rapport à la nature joue lui aussi un rôle fondamental dans la pensée des hommes, celle-ci étant à la fois la cause et l'effet de ce rapport à la nature. Cette conception se retrouve chez Michelet, dont le principe méthodologique est formulé en ces termes:

Une critique nouvelle commence, écrit-il dans une note de la Bible de I'humanité, plus forte et plus sérieuse. Les religions, si profondément étudiées aujourd'hui, ont été subordonnées au genius qui les fit, à leur créatrice, l'âme, au développement moral dont elles sont le simple fruit. II faut d'abord poser la race avec ses aptitudes propres, les milieux où elle vit, ses mœurs naturelles; alors, on peut l'étudier dans sa fabrication des dieux, qui, à leur tour, influent sur elle. C'est le circulus naturel. ${ }^{44}$

Pour montrer comment cette vision ethnodifférentialiste fonctionne dans le discours métapolitique micheletien, je rappellerai un point essentiel de sa conception de la régénération sociale : tout se joue au niveau de la famille. Or, selon I'historien, le problème de la société de son temps est que la « famille » conserve une structure chrétienne. Procédant de la matrice syro-juive, elle serait déséquilibrée, fondée sur l'intérêt et déstabilisée par l'intrusion du prêtre. Dans son projet de régénération spirituelle de la société, l'historien oppose à la tradition judéo-chrétienne de la famille une autre tradition, la tradition familiale issue des Aryâs primifs - « nos ancêtres légitimes »: la famille des Védas. Ainsi Michelet décrit-il le couple sémitique :

Ces Moloch, ces cruels marchands [...] n'avaient que faire des Syriennes. Celles-ci étaient des veuves. [...] Elles rêvaient. Et jamais il n'y eut de si puissantes rêveuses. La Parthéno-Génèse, la force du désir

43 RENAN, Ernest, (1859), Le Livre de Job, étude sur l'âge et le caractère du poëme, Paris, Michel Lévy Frères, réédition de 1859, p. XX de la préface.

44 MICHELET, Bible de l'humanité, note 1, p. 64. 
qui sans mâle est féconde, éclata dans la Syrienne en deux enfants qu'elle fit seule : I'un est le Messie-femme, qui a délivré Babylone, serve jusque-là de Ninive, la grande Sémiramis, née poisson, devenue colombe, qui épouse toute la terre, finit par épouser son fils. L'autre est un dieu de deuil, le Seigneur (Adonaï ou Adonis). Il est né de l'inceste, et son culte mêlé de pleurs, d'amour, tient de l'inceste encore. La grande légende syrienne [...] aboutit à cette création féminine d'immense importance, Adonis, mort, ressuscité. Culte sensuel et pleureur, par lequel le monde descendit misérablement sur la pente de l'énervation. ${ }^{45}$

À cette vision du couple (un tyran impuissant et une vierge achetée), Michelet oppose le type védique de la famille, repérable aussi selon lui chez ses fils, à savoir dans l'Inde ancienne, dans la Perse mazdéenne et dans la Grèce pré dorienne. II écrit à son propos : "Que lisons-nous dans la genèse vénérable des Aryâs, dans les hymnes de leur Rig-Véda, incontestablement le premier monument du monde? Deux personnes unies, I'homme, la femme, d'un élan commun, remercient la lumière, chantent ensemble un hymne à Agni ». ${ }^{46}$ Toutes les dimensions de ce que Michelet invoque comme la tradition aryenne de la famille sont présentes : un culte domestique du feu où l'époux et l'épouse sont dans une relation d'égalité procédant du consentement mutuel ; il en allait de même en Grèce, avant l'invasion du dorisme, où la famille traditionnelle était « cette famille naturelle et sainte qu'on voit dans les Védas, qu'on voit dans l'Avesta ${ }^{4}{ }^{47}$

Le schéma logique est en place : il conduit à écarter purement et simplement la tradition sémitique contre-nature de la Grâce au profit de la tradition «naturelle » de la future République dont la condition onto théologique est de substituer "nos » Bibles naturalistes et fécondes à la Bible hébraïque contre-nature et stérile. Ces origines indo-européennes sont conçues comme une source, ou plus précisément « notre » source, bref comme une réactivation d'une identité oubliée, au prix d'une confusion temporelle entre l'antique et le moderne:

Pour le foyer surtout et les affections du cœur, pour les idées élémentaires de travail, de droit, de justice, la haute antiquité, c'est nous. L'Inde primitive des Védas, I'Iran de l'Avesta, qu'on peut nommer l'aurore

45 Ibid., pp. 313-314.

46 Ibid., p. 26.

47 Ibid., p. 171. 
du monde, dans les types si forts, si simples et si touchants qu'ils ont laissés de la famille, du travail créateur, sont bien plus près de nous que la stérilité, l'ascétisme du Moyen âge. ${ }^{48}$

\section{DEUX PHÉNOMÈnES INDÉPENDANTS ?}

Au-delà des différences entre les Notions élémentaires de linguistique de Nodier et la Bible de l'humanité de Michelet, ces discours qui se réfèrent aux " langues premières » présentent une homogénéité qui réside dans la manière dont la pensée du social se voit ordonnée à l'enquête ethnolinguistique. Dans les deux cas, l'origine révélée par l'étude des langues premières est conçue non comme un lieu primitif d'où l'histoire s'éloignerait mais comme une source, un dévoilement, voire comme ce qui donne sens au social. L'origine devient l'originaire, non plus ce qui est « informe » mais au contraire ce qui a formé une manière d'être propre à un groupe humain et dont l'éloignement est cause de perte de sens. C'est ce « brouillage de temporalités ${ }^{49}$ selon la formule de Bruno Karsenti qui rend possible la mise en équation de l'origine (antique) et de l'identité (moderne) : I'identité se révèle dans "l'originaire » et c'est à cette source que le social se ressource, ces origines représentent, pourrait-on dire, ses « ressources ». Car cette origine n'est pas le lieu où l'on pensait moins bien : on y pensait autrement, voire mieux. L'antique est alors considéré comme un rapport « premier » au monde, un rapport « vrai » dont la rationalité nous aurait éloigné.

Dans les deux cas également, l'identité d'un peuple coïncide avec la particularité d'un passé : mon père me parle dans la langue de son petit pays et c'est ce rapport originaire au monde qui me confère mon identité de sujet parlant le patois, langue native par opposition à la langue moderne qui serait quant à elle une langue pauvre en sens car remplie de « cette multitude $\mathrm{d}^{\prime}$ argotismes à demi sauvages et à demi présomptueux qui débordent du dictionnaire des nations civilisées $» .50$

48 Ibid., p. III. Pour davantage de précision concernant l'usage de la philologie aryaniste de Michelet évoqué dans le présent passage, je me permets de renvoyer à mon ouvrage : ARAMINI, Aurélien (2013), Michelet, à la recherche de l'identité de la France, De la fusion nationale au conflit des traditions, Besançon, Presses Universitaires de Franche-Comté, coll. AGON.

49 KARSENTI, Bruno (2009), "De I'historiographie ancienne à la science sociale : une nouvelle lecture de La Cité antique de Fustel de Coulanges», p. 6, in FUSTEL DE COULANGES, Numa Denis La cité antique, Paris, Flammarion.

50 NODIER, Notions élémentaires de linguistique, p. 251. 
Enfin, les références aux langues premières nourrissent des discours à contre-courant de l'idée de progrès qui implique l'existence de sauts qualitatifs irréversibles: Nodier et Michelet expriment tous deux une fascination vis-à-vis d'un passé considéré comme porteur d'une vérité que le monde moderne a oublié et qu'il faut retrouver. Sous leurs plumes, I'avenir national se ressource dans les langues premières.

Ces trois points communs repérables dans bien d'autres discours mobilisant la référence aux langues premières durant les deux premiers tiers du XIXe siècle, vont être l'objet de fortes critiques de la part d'auteurs emblématiques de la Troisième République et tout particulièrement du "second » Renan $^{51}$ et de Fustel de Coulanges. Au-delà de la diversité des ressorts de leur critique, ces auteurs me semblent s'inscrire dans la dynamique critique vis-à-vis des langues premières qui aboutit au positionnement républicain et à une certaine conception de la nation pour laquelle l'appartenance politique n'est plus déterminée par l'ancrage dans les mondes auxquels les langues premières donnent accès.

\section{MUtATIONS DES Discours :}

\section{historiographIE de LA CITÉ ANTIQUe et PHILOSOPHIE dE LA NATION}

Dans un corpus de discours toujours singuliers mais qui possèdent cependant une homogénéité théorique, deux textes, à des niveaux différents, justifient de parler de «mutations » dans les discours sur les langues premières : la Cité antique de Fustel de Coulanges et les deux lettres de Renan écrites en réponse au savant allemand David-Frédéric Strauss. S'il y a mutation, c'est parce que ces discours mobilisent encore la référence aux langues premières tout en renouvelant en profondeur la manière dont vont être prises en charge les « langues premières » dans la perspective de penser le social et la nation. Dans cette perspective dynamique, s'equisse une certaine pensée républicaine de la nation qui va pouvoir s'affranchir de la tentation de chercher dans les supposées langues premières le fondement $d$ 'une appartenance nationale.

\section{L'historiographie de la Cité antique selon Fustel}

En 1864, sont publiés deux textes dont la mise en regard rend particulièrement manifeste une mutation du discours historiographique mobilisant les antiquités aryennes: la Bible de l'humanité d'un Michelet vieillissant et La Cité antique du jeune Fustel de Coulanges. L'un et l'autre mobilisent la référence indo-européenne dans leur stratégie argumentative : le premier

51 Concernant l'évolution intellectuelle de Renan, voir la dernière partie du présent article. 
pour penser la tradition religieuse légitime de la cité de l'avenir et l'autre pour penser la croyance organisatrice et régulatrice de la " cité antique ». Cependant, une différence majeure apparaît entre les deux historiens quant à l'usage de cette référence.

Michelet cherche dans les antiquités aryennes la source où puiser le credo d'une cité de l'avenir. À l'inverse, le futur représentant emblématique de l'histoire méthodique insiste sur la distance infranchissable entre les croyances communes aux trois grands peuples indo-européens et l'époque présente. Pour Fustel, l'étude des antiquités aryennes est utile pour mieux comprendre les institutions de la cité qui reposent sur le culte des morts en Inde, à Rome et en Grèce ancienne : ${ }^{52}$ "Ces idées et ces rites sont ce qu'il y a de plus vieux dans la race indo-européenne, et sont aussi ce qu'il y a eu de plus persistant $» .^{53}$ Cette étude ne saurait toutefois être mise au service $d^{\prime}$ une régénération religieuse de la cité du présent : aussi Fustel précise-t-il qu'il faut étudier ces antiquités indo-européennes "sans songer à nous ». ${ }^{54}$ Comme l'explique Bruno Karsenti, il ne s'agit pas seulement d'une précaution contre une forme de subjectivité déplacée qui projetterait sur le passé ses propres vues. ${ }^{55} C^{\prime}$ est une mise en garde beaucoup plus forte : cette origine indo-européenne ne fait plus sens pour notre présent.

La question essentielle est de savoir pourquoi elle ne fait plus sens pour nous. Trois types de réponses figurent dans le texte, correspondant chacun à un pallier argumentatif. Une première réponse consiste à dire qu'une autre croyance - le christianisme - s'est substituée à cette ancienne croyance. Cette réponse est insuffisante car cette nouvelle croyance ne s'est substituée à l'ancienne que parce que celle-ci a décliné. La deuxième réponse s'intéresse au contenu même de l'antique croyance. Pourquoi a-t-elle décliné ? Elle ne concernait qu'une " association humaine [qui] était nécessairement bornée dans une certaine circonférence autour d'un prytanée, et où l'on ne voyait pas la possibilité de fonder des sociétés plus grandes $»{ }^{56}$ Mais là encore : le changement de l'association humaine et du type de société est-il la cause ou l'effet du changement dans la croyance?

D'où cette question essentielle qui correspond au troisième niveau de réponse : qu'est-ce qui permet de comprendre ce qui a changé dans cette

52 Cf. le deuxième chapitre du premier livre consacré au « culte des morts », FUSTEL DE COULANGES, La Cité antique, p. 47.

53 Ibid., p. 49.

54 Ibid., p. 34.

55 KARSENTI, « De l'historiographie ancienne à la science sociale... ».

56 FUSTEL DE COULANGES, La Cité antique, p. 537. 
croyance en tant que croyance? Ce qui conduit à se demander non seulement pourquoi elle $n^{\prime}$ est plus crue par les anciens mais aussi pourquoi elle n'est plus croyable pour nous autres modernes. Et c'est là qu'intervient la réponse la plus forte pour mesurer l'écart infranchissable entre " eux » et «nous » ou, plus précisément entre « nos pères » et « notre temps ». Fustel répond : ces croyances ne font plus sens car l'intelligence a progressé : à la différence de ce que pense Michelet, nous autres, modernes, ne pensons pas seulement autrement, nous pensons mieux parce que nos progrès intellectuels font que nous ne pouvons plus croire aux absurdités auxquelles croyaient nos pères. En quoi consiste donc la croyance d'où procède la cité antique? C'est la croyance selon laquelle le mort vit encore, qu'il est là vivant d'une certaine manière sous la terre, sous la pierre du foyer domestique, $d$ 'où la nécessité de le nourrir et de le désaltérer. ${ }^{57}$ Après avoir exposé cette croyance, Fustel ajoute que ces idées « paraissent bien fausses et ridicules $»{ }^{58} \|$ l'écrit parce qu'il considère qu'elles le sont effectivement quant à leur contenu quant bien même les anciens les tenaient pour vraies. L'erreur que commet Michelet consiste à penser qu'elles relèvent $d^{\prime}$ 'un autre régime de vérité ou d'une intuition originaire du monde supérieure à la pensée rationnelle et qu'elles pourraient être éventuellement réactivées. Ce que Fustel met en relief c'est le fait que nos pères partageaient ces croyances, quand bien même elles sont effectivement «bien fausses » et « ridicules » et que nous avons raison, nous qui avons progressé, de les considérer comme telles. Dans l'esprit de l'historien, il n'y a pas d'ambiguïté : ces croyances obscures et scientifiquement dépassées affirmaient " l'existence vague et indécise de l'être humain, invisible mais non immatériel, et réclamant des mortels une nourriture et des breuvages ». ${ }^{59}$ Nous ne croyons plus cela ef nous ne pouvons plus le croire car nos connaissances et notre intelligence du monde et de la nature ont progressé : il y a eu un saut qualitatif irréversible. Nous savons que le mort n'a pas besoin de nourriture : ce sont, dit Fustel, le «travail des philosophes » et le " progrès de la pensée » qui ébranlent « les vieux principes de l'association humaine $» .{ }^{60}$ Nous touchons alors ce qui fait la force et la fragilité de la croyance : la croyance n'est pas volontaire, je ne décide pas de croire que mon ancêtre a faim dans sa tombe; les progrès dans la manière de penser $m^{\prime}$ interdisent de tenir pour vrais de tels contenus de croyances. La vraie originalité du propos de

57 Ibid., p. 49.

58 Ibid., p. 46.

59 Ibid., p. 49.

60 lbid., p. 537. 
Fustel - et son apport à une pensée de la croyance - consiste à montrer que ces croyances en la vie des morts étaient cependant tenues pour vraies par nos ancêtres malgré leur absurdité manifeste, absurdité non seulement pour nous mais en soi. C'est donc le «croire » et non l'éventuelle rationalité de ce qui est "cru » qui fait qu'elles ont été efficaces pour nos ancêtres et qu'elles leur ont permis de conserver le type d'association qu'était la " cité antique ». La " cité antique » à laquelle donne accès l'étude des langues premières indo-européennes éclaire la « cité moderne », la « nation », par sa différence : si «dans les vieux âges, la religion et l'État ne faisait qu'un », nous autres modernes, avec le christianisme, avons séparé "la religion du gouvernement $» .{ }^{61}$ La recherche d'une régénération "spirituelle » dans les antiquités indo-européennes $n^{\prime}$ a donc aucun sens pour une nation à venir car nous ne pouvons plus croire en ce que crurent les hommes de la cité antique ${ }^{62}$ et nous ne pouvons encore moins nous rassembler, comme nos ancêtres, autour d'une telle croyance. Nous n'avons donc plus $d^{\prime}$ « héritage » indo-européen.

\section{Philologie et pensée de la nation : le débat entre Renan et Strauss}

La réfutation de l'actualisation de l'originaire se manifeste, selon une modalité différente, dans le cadre de la pensée de la nation chez Renan qui fut justement l'un des théoriciens de la vision ethnodifférentialiste durant la décennie précédente. Dans ses deux Lettres à Strauss, ${ }^{63}$ la première écrite durant la guerre de 1870 et la seconde après la défaite et l'annexion de I'Alsace Lorraine par l'Allemagne, il aborde une thématique qui anticipe en partie seulement sur la conférence Qu'est-ce qu'une nation? prononcée à la Sorbonne le 11 mars 1882 : le centre du propos de Renan consiste en une critique de l'usage de la philologie « locale » pour penser la constitution de la nation. L'enjeu est de montrer que l'identité de la France n'est pas une

61 lbid., pp. 542-543.

62 Ce n'est d'ailleurs pas un hasard si un ensemble de groupuscules nationalistes antirépublicains se réfère à une tradition " ancestrale » puisant dans les supposées sources indo-européennes de la culture "française ». Sur ce point, on consultera avec profit l'ouvrage de FRANÇOIS, Stéphane (2014), Au-delà des vents du nord, L'extrême droite française, le pôle Nord et les Indo-Européens, Lyon, Presses universitaires de Lyon, qui étudie tout particulièrement le cas du GRECE.

63 Ces lettres ont fait l'objet d'une réédition par Joël Roman dans le volume RENAN, Ernest (1992), Qu'est-ce qu'une nation? et autres essais politiques, textes choisis et présentés par Joël Roman, Paris, Presses Pocket, p. 107 sqq. 
donnée - naturelle ou historiquement première - mais une construction morale à partir d'une diversité initiale. S'esquisse alors une certaine conception de la nation pour laquelle ne fait plus sens l'ancrage dans les mondes humains sédimentés dans les langues premières.

Dans sa seconde lettre à Strauss, Renan oppose deux conceptions de la nation : ${ }^{64}$ la conception allemande et la conception française. D'une part, la conception allemande : "la politique ethnographique et archéologique », la « politique des races ». D'autre part, la conception française : "la politique libérale », la " politique du droit des nations ». ${ }^{65}$ Pour la question de l'appartenance de l'Alsace à la France, l'argument de Strauss consiste à affirmer qu'elle est ethniquement et linguistiquement germanique ou plutôt, qu'elle est considérée comme ethniquement germanique parce que linguistiquement germanique. Mais l'argument va plus loin dans la deuxième lettre de Strauss : en France, la nature germanique de l'Alsace ne peut être qu'opprimée alors qu'en Allemagne, elle pourra s'exhausser, bref I'adhésion morale viendra d'elle-même sur le fondement de son appartenance naturelle.

La force de l'argument que Renan oppose à Strauss réside d'abord dans le fait qu'il ne nie pas l'identification de la langue et de la provenance ethnique. Oui, écrit-il à ce dernier, l'Alsace est « allemande de race et de langue $»{ }^{66}$ Mais l'opération théorique effectuée par Renan consiste à découpler la recherche de l'origine - dont les archives sont les langues - de l'identité nationale actuelle; or, la pensée française, de la Restauration à la fin du Second Empire, s'était régulièrement engagée dans la voie inverse, comme l'avait fait l'Allemagne. C'est justement cet usage de la philologie répandu dans les deux premiers tiers du XIXe siècle français - que le Renan d'après la défaite de 1870 récuse dans la seconde de ses lettres à Strauss : «Défiez-vous donc de l'ethnographie ou plutôt ne l'appliquez pas trop à la politique. Sous prétexte d'une étymologie germanique, vous prenez pour la Prusse tel village de Lorraine $» ?^{67}$

L'argumentation mobilisée par Renan fonctionne en deux temps. Elle s'appuie $d^{\prime}$ abord sur un argument qui montre que l'archéologie philologique du peuple conduit à une thèse absurde :

64 Pour une lecture nuancée de l'opposition entre ces deux conceptions de la nation, l'une française et spirituelle, l'autre allemande et raciale, voir l'article de VENAYRE, Sylvain (2017) : " 1882 Professer la nation », dans BOUCHERON, Patrick (ed.) : Histoire mondiale de la France, Paris, Éditions du Seuil, p. 520.

65 RENAN, « Nouvelle lettre à M. Strauss », p. 157.

66 Ibid., p. 156.

67 Ibid., p. 158-159. 
Presque partout où les patriotes fougueux de l'Allemagne réclament un droit germanique, nous pourrions réclamer un droit celtique antérieur, et, avant la période celtique, il y avait, dit-on, les Allophyles, les Finnois, les Lapons ; et avant les Lapons, il y eut les hommes des cavernes, il y eut les orangs-outans. Avec cette philosophie de l'histoire, il n'y aura de légitime que le droit des orangs-outans, injustement dépossédés par la perfidie des civilisés. ${ }^{68}$

L'archéologie linguistique du peuple serait ainsi une absurdité théorique et l'idée de "langue native » dont parlait Nodier une erreur de perspective historique sur des langues qui sont toujours nécessairement historiques. La régression à une langue première voire à une langue " naturelle " précédant l'histoire est vouée à l'échec. Et, même si une telle régression était possible, ces mondes archaïques sont définitivement engloutis : Renouvier écrira en ce sens que " la guerre et la mort ont détruit les vraies races, les vraies mœurs, les vraies religions, les vraies langues premières, et les nations $d$ 'ordre naturel $»{ }^{69}$ Dès lors, la pensée de la nation républicaine s'affranchit de la référence aux langues premières et aux univers de sens qui se sont sédimentés en elles.

Si l'appartenance ne se trouve pas dans l'origine ethnique révélée par la philologie, qu'est-ce que l'identité nationale ? Si je suis constitué par l'histoire, il semblerait pourtant évident que la langue, trace de l'histoire, doive révéler mon identité. Or, et c'est justement le deuxième temps de l'argumentation, Renan récuse cette relation de conséquence entre l'ethnolinguistique et l'identité. Son argumentation conduit à affirmer que la nation se fonde non sur la nature première qui est introuvable mais sur un " consentement actuel ", sur la « volonté qu'ont les différentes provinces d'un État de vivre ensemble ${ }^{70}$ retrouvant finalement l'idée $d^{\prime}$ un acte par lequel un peuple est un peuple qui ne saurait se déduire de ses éléments ethnolinguistiques. $C^{\prime}$ est dans cette perspective que Renouvier invoquera, contre l'idée de nationalité naturelle, un contrat social explicite ou implicite au fondement de la République. l'identité se construit avant tout dans le présent d'un vouloir : de la "nature » donnée à la «nation » voulue, il y a un saut qualitatif. Ainsi la nation n'est-elle pas naturelle mais morale. Toutefois, il ne s'agit pas de penser la nation comme un déracinement ou un volontarisme niant I'histoire. L'acte fondateur du peuple n'est pas anhistorique et je conclurai

68 Ibid., p. 155.

69 RENOUVIER, Charles (1869), Science de la morale, Paris, Ladrange, t. II, p. 421.

70 RENAN, « Nouvelle lettre à M. Strauss », p. 156. 
sur ce point, en examinant la formule précise utilisée par Renan dans sa lettre pour affirmer que I'Alsace est française : il invoque « le droit qu'ont les Alsaciens, êtres vivants en chair et en os, de n'obéir qu'à un pouvoir consenti par eux ». Le texte n'évoque pas des citoyens indéfinis historiquement $d^{\prime}$ une part et le peuple français d'autre part : il est question $d^{\prime}$ " Alsaciens ", c'est-à-dire d'êtres identifiés explicitement par leur appartenance particulière. Renan ne nie pas le fait que le consentement individuel est situé « ethniquement » en reconnaissant dans sa formulation l'existence d'un groupe humain intermédiaire entre l'individu et le peuple, groupe dont l'existence semblait justement, pour Barère ou Grégoire, interdire de penser la République dans son unité. II ne faut pas voir toutefois dans cette identification « ethnolinguistique » une inconséquence ; c'est même plutôt le ressort de l'argumentation de Renan car, dans sa lettre de 1871, ce sont bien des «Alsaciens » qui consentent, c'est-à-dire des êtres de chair et de sang, de race et de langue allemande, qui expriment pourtant, au-delà de leur race et de leur langue, un consentement actuel à être citoyen français : ce consentement moral n'est contradictoire qu'en apparence avec leur appartenance ethnolinguistique parce qu'en réalité le politique n'est pas la continuation de l'ethnolinguistique. S'ouvre alors la possibilité de penser une " appartenance » politique libérée de " l'ancrage ". ${ }^{71}$ La nation républicaine instituée devra se construire au lieu de se continuer et cette construction se fera, selon les mots de Renouvier, avec des « hommes de toutes races, langues et croyances $\gg .^{72}$

Les dispositifs théoriques de Fustel et de Renan expriment l'exigence d'inscrire dans le devenir des sociétés humaines un principe de rupture entre des mondes humains temporellement distants et sémantiquement hétérogènes entre l'ancien et le moderne; entre l'ethnique et le national - ; il s'agit dans les deux cas de réfuter la confusion entre ces niveaux qui est, au cœur, de

71 Sur ce point, on remarquera que la formule de Qu'est-ce qu'une nation ? apparemment très semblable à celles des lettres à Strauss, est en réalité beaucoup plus claire et tranche la difficulté : «Une province, pour nous, ce sont ses habitants; si quelqu'un en cette affaire a droit d'être consulté, c'est l'habitant» (RENAN, Qu'est-ce qu'une nation??, p. 55). De l'« Alsacien » à l'« habitant», il y a une rupture théorique : I'habitant est situé géographiquement mais non historiquement dans une provenance ethnique. "L'habitant», c'est l'occupant d'un espace, $I^{\prime}$ «Alsacien », quant à lui, renvoie à l'inscription dans une histoire qui a façonné la géographie et les hommes.

72 RENOUVIER, Science de la morale, p. 418. 
certains discours sur les langues premières. Contrairement à ce que disait Nodier, la langue du père n'est pas l'âme du peuple : le principe spirituel qui fait que le peuple vit actuellement, c'est sa volonté. Contrairement à ce que pense Michelet, ce n'est pas la redécouverte de la tradition de nos prétendus ancêtres qui peut produire le consentement actuel assurant l'unité nationale car nous ne pouvons plus tenir pour vraie une telle tradition. Ainsi, l'étude des discours sur les langues premières de la Restauration à l'avènement de la Troisième République permet de mettre au jour les tensions théoriques et le travail conceptuel qui aboutissent à cette thèse fondatrice : les filiations ethnolinguistiques ne fondent pas le social et la création effective d'une « identité » républicaine au sein de la nation n'est pas l'actualisation des identités particulières procédant de l'origine linguistique, qu'elle fasse signe vers la province ou vers un passé archaïque.

\section{BiblographIE}

ARAMINI, Aurélien (2013), Michelet, à la recherche de l'identité de la France, De la fusion nationale au conflit des traditions, Presses Universitaires de Franche-Comté, Besançon, coll. AGON.

ARAMINI, Aurélien (2014), "L'Archéologie linguistique du pouvoir et du peuple chez Augustin Thierry », Revue d'Histoire du XIXe Siècle, $n^{\circ} 49$, 2014/2, pp. 177-191.

ARAMINI, Aurélien (2014), "Zoroastre républicain Les enjeux philosophiques et politiques de la découverte des textes "zends" : Eugène Burnouf et Jean Reynaud», Klesis, 30, pp. 27-54.

BALIBAR, Renée (1985), L'Institution du français Essai sur le colinguisme des Carolingiens à la République, PUF, Paris, coll. "Pratiques théoriques". BRANCA-ROSOFF, Sonia (1999) « Normes et dialectes» in AUROUX, Sylvain (dir.), Histoire des idées linguistiques, t. III « L'hégémonie du comparatisme », Mardaga, coll. "Philosophie et langage », pp. 45-53.

BRUCE-WHYTE, A. (1841), Histoire des langues romanes et de leur littérature depuis leur origine jusqu'au XIVE siècle en trois tomes, Treuttel et Würtz, Paris. BURNOUF, Eugène (1833), "De la langue et de la littérature sanscrite », Revue des deux mondes, livraison du 1 er février 1833, pp. 3-4.

CERTEAU, Michel de, JULIA, Dominique et REVEL, Jacques (2002), Une Politique de la langue, Éditions Gallimard, collection « folio histoire », première édition 1975.

DELAMÉTHERIE Jean-Claude (1806), Considérations sur les êtres organisés : de la perfectibilité et de la dégénérescence des êtres organisés, Courcier, Paris. 
DEMOULE, Jean-Paul (2014), Mais où sont passés les Indo-européens ?, Seuil, Paris.

EDWARDS, William Frederic (1844), Recherches sur les langues celtiques, Imprimerie Royale, Paris.

FALLOT, Samuel (1828), Recherches sur le patois de Franche-Comté, de Lorraine et d'Alsace, Deckherr, Montbéliard.

FOUCAULT, Michel (1966), Les mots et les choses Une archéologie des sciences humaines, Éditions Gallimard, Paris, coll. Tel.

FRANÇOIS, Stéphane (2014), Au-delà des vents du nord, L'extrême droite française, le pôle Nord et les Indo-Européens, Presses Universitaires de Lyon, Lyon.

GUILHAUMOU, Jacques (1986) : «Les Jacobins et la langue provençale : I'initiative linguistique des "missionnaires patriotes" marseillais en $1792 »$, Cahiers Critiques du Patrimoine, Obradors occitan en Provença, 2, p. 11 124.

GUILHAUMOU, Jacques, (1989) : La langue politique et la Révolution française. De l'événement à la raison linguistique, Méridiens Klincksieck, Paris. KARSENTI, Bruno (2009), " De I'historiographie ancienne à la science sociale : une nouvelle lecture de La Cité antique de Fustel de Coulanges », p. 6 , in FUSTEL DE COULANGES, Numa Denis La Cité antique, Flammarion. Paris.

KOERNER, Konrad (1989), "Observations of the Sources, Transmission, and Meaning of 'Indo-European' and related Terms in the Development of Linguistics" in KOERNER, Konrad (ed.) Practicing Linguistic Historiography, John Benjamins Publishing Company, Amsterdam \& Philadelphia, pp. 153-180. LASSEN, Christian (1830), "Über Herrn Professor Bopps grammatisches System der Sanskrit-Sprache », Indische Bibliothek III, ed. August Wilhelm Schlegel, Bonn, 1830, pp. 1-113.

LE GONIDEC, Jean-François, (1821), Dictionnaire breton-français, Angoulême.

LÉCLUSE, Fleury de (1826), Grammaire basque, Toulouse.

LORRAIN Paul (1837), Tableau de l'instruction primaire en France, Hachette, Paris.

MICHELET, Jules (1864), Bible de l'humanité, Chamerot, Paris.

MICHELET, Jules (1971), Histoire romaine, OEuvres complètes, publiées sous la direction de Paul Viallaneix, Flammarion, Paris, t. II.

NODIER, Charles, (1934), Notions élémentaires de linguistique, Renduel, Paris. 
OLENDER, Maurice (1989), les Langues du paradis, Aryens et sémites : un couple providentiel, Éditions du Seuil, Paris, coll. « Hautes Études».

PICTET, Adolphe (1837), De l'affinité des langues celtiques avec le sanscrit par, Duprat, Paris.

PLÖTNER, Bärbel (1994), «Langue littérature et identités nationales et régionales Jacob Grimm entre la France et l'Allemagne Le cas breton » in Espagne, Michel et Werner Michael (dir.), Qu'est-ce qu'une littérature nationale? Approche pour une théorie interculturelle du champ littéraire, Éditions de la Maison des Sciences de l'Homme, Paris, pp. 21 2-234.

RABAULT-FEUERHAHN, Pascale (2008), L'archive des origines. Sanskrit, philologie, anthropologie dans l'Allemagne du XIXe siècle, Cerf, Paris.

RENAN, Ernest (1992), Qu'est-ce qu'une nation? et autres essais politiques, textes choisis et présentés par Joël Roman, Presses Pocket, Paris, $p$. 107 sqq.

RENAN, Ernest, (1859), Le Livre de Job, étude sur l'âge et le caractère du poëme, Michel Lévy Frères, Paris, réédition de 1859.

RENOUVIER, Charles (1869), Science de la morale, Ladrange, Paris, t. II, p. 421.

SCHLIEBEN-LANGE, Brigitte (1999), "La Révolution française », première section du premier chapitre "Luttes sociales, dialectes, contraintes ", in AUROUX, Histoire des idées linguistiques, pp. 23-34.

THIERRY, Augustin (1825), Histoire de la Conquête de l'Angleterre par les Normands, Firmin Didot, Paris.

THIERRY, Augustin (1827), Lettres sur I'histoire de France, Sautelet, Ponthieu et compagnie, Paris.

VENAYRE, Sylvain (2017) : « 1882 Professer la nation », dans BOUCHERON, Patrick (ed.) : Histoire mondiale de la France, Éditions du Seuil, Paris, p. 520.

WEBER, Eugen (1983), La fin des terroirs La modernisation de la France rurale 1870-1914, Fayard, Paris. 